\title{
Choosing Nonmortars: Does it Influence the Performance of FETI-DP Algorithms?
}

\author{
Dan Stefanica \\ City University of New York, Baruch College, Department of Mathematics
}

\begin{abstract}
Summary. We investigate whether different choices of nonmortar sides for the geometrically conforming partitions inherent to FETI-DP influence the convergence of the algorithms for four different preconditioners. We conclude experimentally that they do not, although better condition number estimates exist for a NeumannDirichlet choice of nonmortars.
\end{abstract}

\section{Introduction}

The dual-primal FETI (FETI-DP) method is an iterative substructuring method using Lagrange multipliers. It was introduced in Farhat et al. [1999] for two dimensional problems as a FETI-type algorithm which does not require solving singular problems on individual subdomains, and was extended to three dimensional problems in Farhat et al. [2000] and Klawonn et al. [2002]. The scalability and optimal convergence properties of FETI-DP were established in Mandel and Tezaur [2001] and Klawonn et al. [2002].

Mortar finite elements were first introduced by Bernardi et al. [1994] and are actively used in practice for their advantages over the conforming finite elements, e.g., flexible mesh generation and straightforward local refinement. Extending FETI and FETI-DP algorithms to mortar discretizations is a natural idea and such work can be traced back to Lacour and Maday [1997]. Most of this work was computational, investigating the convergence properties of various FETI preconditioners for mortar algorithms; see, e.g., Stefanica [2001, 2002]. Condition number estimates were established for two FETI-DP preconditioners for mortar methods by Dryja and Widlund [2002a,b]. Recently, similar bounds were obtained by Dryja and Proskurowski [2004] for problems with discontinuous coefficients.

The FETI-DP algorithms discussed here are based on geometrically conforming partitions of the computational domain. Across every subdomain side there is exactly one edge belonging to a different subdomain. For mortar methods, either one of these sides is chosen to be a nonmortar, with the other one 
being a mortar side. In Dryja and Widlund [2002a], the condition number estimate for a FETI-DP preconditioner depends on whether the choice of nonmortars is random or is made according to a Neumann-Dirichlet ordering of the subdomains, i.e., with the sides of any subdomain being either all mortars or all nonmortars.

In this paper, we investigate experimentally this result and conclude that, in practice, the special choice of nonmortars does not influence the numerical convergence of the FETI-DP algorithm. We also compare the numerical performance of three other possible preconditioners, a generalized one suggested in Dryja and Widlund [2002b] and two other similar to the Dirichlet and generalized preconditioners for FETI mortar algorithms, and conclude that the generalized preconditioners have the best convergence properties.

The notations in this paper are related to those of Dryja and Widlund [2002b] and of Farhat et al. [1999]. For details omitted here due to space constraints, we refer the reader to the same two papers.

\section{Mortar Finite Element Spaces of First Type}

To construct the mortar finite element space $W$, the computational domain $\Omega$ is partitioned into nonoverlapping rectangular subdomains $\Omega_{i}, i=1: N$. For the FETI-DP algorithms considered here, the partition must be geometrically conforming, i.e, the intersection between the closures of any two subdomains is either empty, or consists of a vertex or an entire edge, and the mortars must be of the first type, i.e., continuity is required at the corner nodes.

Across the interface $\Gamma$, i.e., the set of points that belong to the boundaries of at least two subregions, we do not require pointwise continuity. Since the partition is geometrically conforming, the edges of the subdomains are pairwise opposite. From each pair, one edge, denoted by $\delta_{m(i)}$ and assumed to belong to the subdomain $\Omega_{i}$, is chosen to be a nonmortar side, while the other edge, denoted by $\gamma_{m(j)}$ and belonging to $\Omega_{j}$, is a mortar side.

The restriction of a mortar function $v \in W$ to any subdomain is a $P_{1}$ or a $Q_{1}$ finite element function. We assume that each subdomain $\Omega_{i}$ has a diameter of order $H$ and that its triangulation has a mesh size of order $h$. Let $v_{i}$ and $v_{j}$ be the restrictions of $v$ to an arbitrary nonmortar side $\delta_{m(i)}$ and to its opposite mortar side $\gamma_{m(j)}$, respectively. Then $v_{i}$ and $v_{j}$ have the same values at the left and right end points of $\delta_{m(i)}$ and $\gamma_{m(j)}$, respectively, and the following mortar conditions have to be satisfied:

$$
\int_{\delta_{m(i)}}\left(v_{i}-v_{j}\right) \psi d s=0, \quad \forall \psi \in M\left(\delta_{m(i)}\right),
$$

where $M\left(\delta_{m(i)}\right)$ is a space of test functions having the same dimension as the number of interior nodes of $\delta_{m(i)}$, i.e., piecewise linear functions on $\delta_{m(i)}$ which are constant in the first and last mesh interval. 
For algorithmic purposes, we derive a matrix formulation for the mortar conditions (1). Let $v_{i, r}$ be the vector of interior nodal values of $v_{i}$ on $\delta_{m(i)}$, and let $v_{i, c}$ be the vector of corner nodal values of $v_{i}$ on $\delta_{m(i)}$. We define $v_{j, r}$ and $v_{j, c}$ similarly for $v_{j}$ on $\gamma_{m(j)}$. The matrix formulation of (1) is

$$
B_{\delta_{m(i)}, r} v_{i, r}+B_{\delta_{m(i)}, c} v_{i, c}-B_{\gamma_{m(j)}, r} v_{j, r}-B_{\gamma_{m(j)}, c} v_{j, c}=0,
$$

where the matrix $B_{\delta_{m(i)}, r}$ is banded for classical mortars and equal to the identity for the biorthogonal mortars of Wohlmuth [2000].

\section{FETI-DP Algorithms for Mortars}

As model problem we choose the Poisson problem with mixed boundary conditions on $\Omega$. Given $f \in L^{2}(\Omega)$, find $u \in H^{1}(\Omega)$ such that

$$
-\Delta u=f \text { on } \Omega, \quad \text { with } u=0 \text { on } \partial \Omega_{D} \text { and } \partial u / \partial n=0 \text { on } \partial \Omega_{N},
$$

where $\partial \Omega_{N}$ and $\partial \Omega_{D}$ are the parts of $\partial \Omega=\partial \Omega_{N} \cup \partial \Omega_{D}$ where Neumann and Dirichlet boundary conditions are imposed, respectively,

To discretize (3), let $W_{i}$ be the restriction to $\Omega_{i}$ of the mortar finite element space $W$. The primal variables space is $\widehat{W}$, the subspace of $\Pi_{i=1}^{n} W_{i}$ of functions continuous at each corner node. Lagrange multipliers are used to enforce the mortar conditions (1). The dual variables space is $\Pi_{m} M\left(\delta_{m}\right)$, where the product is considered over all the nonmortar sides. We partition the nodal values of $v \in \widehat{W}$ into the corner nodal values $v_{c}$ and the remainder nodal values $v_{r}$. Note that $v_{r}$ can be further split into interior nodal values $v_{\text {int }}$ and remainder boundary nodal values $v_{b_{r}}$. The continuity conditions at the subdomain corners are enforced by using a global vector of degrees of freedom $v_{c}^{g}$ and a global to local map $L_{c}$ with one nonzero entry per line equal to 1 , and by requiring that $v_{c}=L_{c} v_{c}^{g}$. Therefore, $v=\left[v_{i n t} ; v_{b_{r}} ; v_{b_{c}}\right]=\left[v_{r} ; v_{b_{c}}\right]=\left[v_{r} ; L_{c} v_{c}^{g}\right]$.

Let $K$ be the stiffness matrix of the discrete problem and let $K_{r r}, K_{r c}$, and $K_{c c}$ be its blocks corresponding to a decomposition of $v$ into $v_{r}$ and $v_{c}$. We use a Lagrange multiplier matrix $B$ to enforce the mortar conditions (1). The matrix $B$ has one horizontal block, $B_{\delta_{m(i)}}$, for each nonmortar side $\delta_{m(i)}$, built from the columns of $B_{\delta_{m(i)}, r}, B_{\delta_{m(i)}, c}, B_{\gamma_{m(j)}, r}$, and $B_{\gamma_{m(j)}, c}$, with all the other entries zero; cf. (2). We can also write $B=\left[\begin{array}{ll}B_{r} & B_{c}\end{array}\right]$ using vertical blocks corresponding to the remainder and corner nodes.

The saddle point formulation of the model problem is

$$
\left[\begin{array}{ccc}
I & 0 & 0 \\
0 & L_{c}^{T} & 0 \\
0 & 0 & I
\end{array}\right]\left[\begin{array}{ccc}
K_{r r} & K_{r c} & B_{r}^{T} \\
K_{r c}^{T} & K_{c c} & B_{c}^{T} \\
B_{r} & B_{c} & 0
\end{array}\right] \quad\left[\begin{array}{ccc}
I & 0 & 0 \\
0 & L_{c} & 0 \\
0 & 0 & I
\end{array}\right] \quad\left[\begin{array}{c}
u_{r} \\
u_{c}^{g} \\
\lambda
\end{array}\right]=\left[\begin{array}{c}
f_{r} \\
L_{c}^{T} f_{c} \\
0
\end{array}\right]
$$

After eliminating the primal variables $u_{r}$ and $u_{c}^{g}$ we obtain the dual problem

$$
F_{I_{r r}}+\widetilde{F}_{I_{r c}}\left(K_{c c}^{*}\right)^{-1} \widetilde{F}_{I_{r c}}^{T} \lambda=d
$$


where $F_{I_{r r}}=B_{r} K_{r r}^{-1} B_{r}^{T}, \widetilde{F}_{I_{r c}}=B_{r} K_{r r}^{-1} K_{r c}-B_{c} L_{c}$, and $K_{c c}^{*}=L_{c}^{T}\left(K_{c c}-\right.$ $\left.K_{c r} K_{r r}^{-1} K_{r c}\right) L_{c}$.

As pointed out by Kim and Lee [2002], the FETI-DP algorithms of Dryja and Widlund are not applied on the mortar space $W$, but on a space very close to $W$. We call this the DW setting throughout the rest of the paper. Using the notations from section 2, the condition (3.4) from Dryja and Widlund [2002b] (the same as (6) from Dryja and Widlund [2002a]) used to build the block of $B$ corresponding to the nonmortar $\delta_{m(i)}$ can be expressed as

$$
B_{\delta_{m(i)}, r} v_{i, r}+B_{\gamma_{m(j)}, r} v_{j, r}=0
$$

which is different from the mortar condition (2). Thus, the discrete problem solved in Dryja and Widlund $[2002 \mathrm{~b}, \mathrm{a}]$ can be written as

$$
\left[\begin{array}{ccc}
I & 0 & 0 \\
0 & L_{c}^{T} & 0 \\
0 & 0 & I
\end{array}\right]\left[\begin{array}{ccc}
K_{r r} & K_{r c} & B_{r}^{T} \\
K_{r c}^{T} & K_{c c} & 0 \\
B_{r} & 0 & 0
\end{array}\right]\left[\begin{array}{ccc}
I & 0 & 0 \\
0 & L_{c} & 0 \\
0 & 0 & I
\end{array}\right]\left[\begin{array}{c}
u_{r} \\
u_{c}^{g} \\
\lambda
\end{array}\right]=\left[\begin{array}{c}
f_{r} \\
L_{c}^{T} f_{c} \\
0
\end{array}\right]
$$

and corresponds to (4) for $B_{c}=0$. The dual problem for the DW setting is

$$
B_{r} \widetilde{S}^{-1} B_{r}^{T} \lambda=\widetilde{d},
$$

where $B_{r} \widetilde{S}^{-1} B_{r}^{T}=F_{I_{r r}}+F_{I_{r c}}\left(K_{c c}^{*}\right)^{-1} F_{I_{r c}}^{T}$, with $F_{I_{r c}}=B_{r} K_{r r}^{-1} K_{r c}$ obtained from $\widetilde{F}_{I_{r c}}$ by setting $B_{c}=0$. We note that our matrix $B_{r}$ is the same as the matrix $B$ from Dryja and Widlund [2002b,a].

Let $S_{r r}=K_{b_{r} b_{r}}-K_{b_{r} \text { int }} K_{\text {int,int }}^{-1} K_{b_{r} \text { int }}^{T}$ be a Schur complement-type matrix. The Dirichlet preconditioner $M_{D}^{-1}$ and the generalized preconditioner $\widetilde{M}_{\text {gen }}^{-1}$ were introduced in Dryja and Widlund [2002b] for the DW setting dual problem (7):

$$
\begin{aligned}
M_{D}^{-1} & =B_{r} S_{r r} B_{r}^{T} ; \\
\widetilde{M}_{\mathrm{gen}}^{-1} & =\operatorname{diag}\left(B_{r} \widetilde{B}_{r}^{T}\right)^{-1} \widetilde{B}_{r} S_{r r} \widetilde{B}_{r}^{T} \operatorname{diag}\left(\widetilde{B}_{r} B_{r}^{T}\right)^{-1} .
\end{aligned}
$$

The generalized matrix $\widetilde{B}_{r}$ is obtained by scaling the $B_{\gamma_{m(j)}, r}$ in the block corresponding to the nonmortar side $\delta_{m(i)}$ by $h_{\delta_{m(i)}} / h_{\gamma_{m(j)}}$; see (3.13) from Dryja and Widlund [2002b] for more details.

In Dryja and Widlund [2002a], it was shown that, for a random choice of the nonmortar sides, $\kappa\left(M_{D}^{-1}\right) \leq C(1+\log H / h)^{4}$, while $\kappa\left(M_{D}^{-1}\right) \leq C(1+$ $\log H / h)^{2}$ if the nonmortar sides are chosen according to a Neumann-Dirichlet ordering, i.e., with all the sides of any subdomain being either all mortars or all nonmortars. In Dryja and Widlund [2002b], it was shown that $\kappa\left(\widetilde{M}_{\mathrm{gen}}^{-1}\right) \leq$ $C(1+\log H / h)^{2}$. All constants denoted by $C$ are independent of $H$ and $h$.

For the dual problem (5), based on the numerical performance of FETI algorithms for mortars, see Stefanica [2001], we suggest the following Dirichlet and generalized preconditioners:

$$
\begin{aligned}
F_{D}^{-1} & =B_{r} S_{r r} B_{r}^{T} \\
F_{\text {gen }}^{-1} & =\operatorname{diag}\left(B_{r} B_{r}^{T}\right)^{-1} B_{r} S_{r r} B_{r}^{T} \operatorname{diag}\left(B_{r} B_{r}^{T}\right)^{-1} .
\end{aligned}
$$




\section{Numerical Results}

We tested the numerical performance of the preconditioners $F_{\text {gen }}^{-1}(11)$ and $F_{D}^{-1}(10)$ for the mortar dual problem (5), and the preconditioners $\widetilde{M}_{\text {gen }}^{-1}(9)$ and $M_{D}^{-1}(8)$ for the approximate dual problem (7).

Our interests were four-fold:

- check the convergence and scalability properties of the resulting algorithms; - compare the performance of the algorithms for mortars to that of the algorithms for the DW setting;

- investigate whether a Neumann-Dirichlet choice of nonmortars improves convergence, in particular for the Dirichlet preconditioner $M_{D}^{-1}$;

- decide which of the four preconditioners performs best.

The model problem was the Poisson equation on the unit square $\Omega$ with mixed boundary conditions. We partitioned $\Omega$ into $N=16,36,64$, and 121 congruent squares, and $Q_{1}$ elements were used in each square. For each partition, the number of nodes on each edge, $H / h$, was taken to be, on average, $5,10,20$, and 40, respectively, for different sets of experiments. The meshes did not match for any neighboring subdomains. The preconditioned conjugate gradient iteration was stopped when the residual norm decreased by a factor of $10^{-6}$. The experiments were carried out in MATLAB.

We report iteration counts, condition number estimates, and flop counts for two different sets of experiments: for randomly chosen nonmortars, in Table 1, and for a Neumann-Dirichlet choice of nonmortars, in Table 2.

Table 1. Convergence results, randomly chosen nonmortars

\begin{tabular}{|c|c|c|c|c|c|c|c|c|c|c|c|c|c|}
\hline & & & en & lized & & Diricl & hlet & DW & Gene & eralized & & V Dir & ichlet \\
\hline$N$ & $H / h$ & Iter & Cond & Mflops & Iter & Cond & Mflops & Iter & Cond & Mflops & Iter & $\overline{\text { Cond }}$ & Mflops \\
\hline 16 & 5 & 7 & 2.6 & $3.0 \mathrm{e}-1$ & 24 & 32 & $1.0 \mathrm{e}+0$ & 8 & 2.3 & $3.3 \mathrm{e}-1$ & 26 & 32 & $1.1 \mathrm{e}+0$ \\
\hline 16 & 10 & 9 & 3.4 & $1.7 \mathrm{e}+0$ & 22 & 41 & $4.3 \mathrm{e}+0$ & 9 & 3.1 & $1.7 \mathrm{e}+0$ & 28 & 40 & $5.4 \mathrm{e}+0$ \\
\hline 16 & 20 & 10 & 4.5 & $1.2 \mathrm{e}+1$ & 23 & 52 & $2.8 \mathrm{e}+1$ & 10 & 4.0 & $1.2 \mathrm{e}+1$ & 30 & 51 & $3.6 \mathrm{e}+1$ \\
\hline 16 & 40 & 11 & 5.7 & $9.5 \mathrm{e}+1$ & 25 & 65 & $2.2 \mathrm{e}+2$ & 10 & 4.9 & $8.6 \mathrm{e}+1$ & 32 & 62 & $2.8 \mathrm{e}+1$ \\
\hline 36 & 5 & 8 & 2.5 & $9.9 \mathrm{e}-1$ & 25 & 31 & $3.1 \mathrm{e}+0$ & 9 & 2.6 & $1.0 \mathrm{e}+0$ & 30 & 33 & $3.5 \mathrm{e}+0$ \\
\hline 36 & 10 & 10 & 3.5 & $5.1 \mathrm{e}+0$ & 26 & 40 & $1.3 \mathrm{e}+1$ & 11 & 3.4 & $5.4 \mathrm{e}+0$ & 32 & 41 & $1.6 \mathrm{e}+1$ \\
\hline 36 & 20 & 12 & 4.5 & $3.5 \mathrm{e}+1$ & 29 & 51 & $8.6 e+1$ & 12 & 4.4 & $3.4 \mathrm{e}+1$ & 35 & 52 & $1.0 \mathrm{e}+2$ \\
\hline 36 & 40 & 13 & 5.7 & $2.7 \mathrm{e}+2$ & 30 & 63 & $6.3 \mathrm{e}+2$ & 13 & 5.5 & $2.6 \mathrm{e}+2$ & 38 & 64 & $7.8 \mathrm{e}+2$ \\
\hline 64 & 5 & 10 & 2.8 & $2.8 \mathrm{e}+0$ & 28 & 36 & $7.9 \mathrm{e}+0$ & 10 & 2.8 & $2.5 \mathrm{e}+0$ & 32 & 37 & $8.0 \mathrm{e}+0$ \\
\hline 64 & 10 & 12 & 3.7 & $1.2 \mathrm{e}+1$ & 29 & 47 & $3.0 \mathrm{e}+1$ & 12 & 3.7 & $1.2 \mathrm{e}+1$ & 37 & 48 & $3.6 \mathrm{e}+1$ \\
\hline 64 & 20 & 13 & 4.8 & $7.1 \mathrm{e}+1$ & 32 & 60 & $1.8 \mathrm{e}+2$ & 13 & 4.8 & $6.9 \mathrm{e}+1$ & 41 & 61 & $2.2 \mathrm{e}+2$ \\
\hline 64 & 40 & 15 & 6.1 & $5.6 \mathrm{e}+2$ & 34 & 76 & $1.3 \mathrm{e}+3$ & 15 & 6.0 & $5.5 \mathrm{e}+2$ & 45 & 76 & $1.7 \mathrm{e}+3$ \\
\hline 121 & 5 & 9 & 2.7 & $6.4 \mathrm{e}+0$ & 29 & 36 & $2.1 \mathrm{e}+1$ & 11 & 3.4 & $6.7 \mathrm{e}+0$ & 37 & 41 & $2.3 \mathrm{e}+1$ \\
\hline 121 & 10 & 12 & 3.7 & $2.8 \mathrm{e}+1$ & 30 & 45 & $7.2 \mathrm{e}+1$ & 13 & 4.5 & $2.8 \mathrm{e}+1$ & 41 & 52 & $8.9 \mathrm{e}+1$ \\
\hline 121 & 20 & 13 & 4.8 & $1.5 \mathrm{e}+2$ & 33 & 61 & $3.8 \mathrm{e}+2$ & 14 & 5.6 & $1.5 \mathrm{e}+2$ & 46 & 68 & $5.0 \mathrm{e}+2$ \\
\hline 121 & 40 & 15 & 6.2 & $1.1 \mathrm{e}+3$ & 36 & 77 & $2.6 \mathrm{e}+3$ & 16 & 6.9 & $1.1 \mathrm{e}+3$ & 51 & 84 & $3.7 \mathrm{e}+3$ \\
\hline
\end{tabular}


Table 2. Convergence results, Neumann-Dirichlet choice for nonmortars

\begin{tabular}{|c|c|c|c|c|c|c|c|c|c|c|c|c|c|}
\hline & & & en & ized & & Diricl & hlet & DW & Gent & eralized & & $V$ Dir & cichlet \\
\hline$N$ & $H / h$ & Iter & Cond & Mflops & Iter & Cond & Mflops & Iter & Cond & Mflops & Iter & Cond & Mflops \\
\hline 16 & 5 & 7 & 2.6 & $3.0 \mathrm{e}-1$ & 20 & 21 & $8.7 \mathrm{e}-1$ & 7 & 2.3 & $2.9 \mathrm{e}-1$ & 22 & 21 & $4.6 \mathrm{e}+0$ \\
\hline 16 & 10 & 8 & 3.5 & $1.6 \mathrm{e}+0$ & 19 & 26 & $3.7 \mathrm{e}+0$ & 8 & 3.1 & $1.5 \mathrm{e}+0$ & 24 & 25 & $4.6 \mathrm{e}+0$ \\
\hline 16 & 20 & 9 & 4.5 & $1.1 \mathrm{e}+1$ & 19 & 33 & $2.3 \mathrm{e}+1$ & 9 & 4.0 & $1.1 \mathrm{e}+1$ & 24 & 32 & $2.9 \mathrm{e}+1$ \\
\hline 16 & 40 & 10 & 5.7 & $8.6 e+1$ & 19 & 42 & $1.7 \mathrm{e}+2$ & 9 & 5.0 & $7.7 \mathrm{e}+1$ & 26 & 40 & $2.3 \mathrm{e}+1$ \\
\hline 36 & 5 & 8 & 2.5 & $9.9 \mathrm{e}-1$ & 25 & 33 & $3.1 \mathrm{e}+0$ & 9 & 2.6 & $1.0 \mathrm{e}+0$ & 28 & 33 & $3.2 \mathrm{e}+0$ \\
\hline 36 & 10 & 10 & 3.4 & $5.1 \mathrm{e}+0$ & 26 & 42 & $1.3 \mathrm{e}+1$ & 11 & 3.5 & $5.4 \mathrm{e}+0$ & 31 & 43 & $1.5 \mathrm{e}+1$ \\
\hline 36 & 20 & 12 & 4.5 & $3.5 \mathrm{e}+1$ & 29 & 54 & $8.6 \mathrm{e}+1$ & 12 & 4.4 & $3.4 \mathrm{e}+1$ & 34 & 54 & $9.8 \mathrm{e}+1$ \\
\hline 36 & 40 & 13 & 5.7 & $2.7 \mathrm{e}+2$ & 31 & 67 & $6.5 \mathrm{e}+2$ & 13 & 5.8 & $2.6 \mathrm{e}+2$ & 37 & 68 & $7.6 \mathrm{e}+2$ \\
\hline 64 & 5 & 9 & 2.7 & $2.5 \mathrm{e}+0$ & 29 & 38 & $8.2 \mathrm{e}+0$ & 10 & 2.9 & $2.5 \mathrm{e}+0$ & 33 & 39 & $8.0 \mathrm{e}+0$ \\
\hline 64 & 10 & 12 & 3.7 & $1.2 \mathrm{e}+1$ & 29 & 49 & $3.0 \mathrm{e}+1$ & 12 & 3.8 & $1.2 \mathrm{e}+1$ & 36 & 49 & $3.5 \mathrm{e}+1$ \\
\hline 64 & 20 & 13 & 4.8 & $7.1 \mathrm{e}+1$ & 30 & 63 & $1.7 \mathrm{e}+2$ & 13 & 4.8 & $6.9 \mathrm{e}+1$ & 42 & 63 & $2.3 \mathrm{e}+2$ \\
\hline 64 & 40 & 15 & 6.2 & $5.6 \mathrm{e}+2$ & 31 & 79 & $1.2 \mathrm{e}+3$ & 15 & 6.1 & $5.5 \mathrm{e}+2$ & 46 & 80 & $1.7 \mathrm{e}+3$ \\
\hline 121 & 5 & 9 & 2.7 & $6.4 \mathrm{e}+0$ & 30 & 40 & $2.2 \mathrm{e}+1$ & 10 & 3.2 & $6.1 \mathrm{e}+0$ & 35 & 38 & $2.2 \mathrm{e}+1$ \\
\hline 121 & 10 & 12 & 3.7 & $2.8 \mathrm{e}+1$ & 31 & 52 & $7.4 \mathrm{e}+1$ & 12 & 4.2 & $2.6 \mathrm{e}+1$ & 39 & 48 & $8.4 \mathrm{e}+1$ \\
\hline 121 & 20 & 13 & 4.8 & $1.4 \mathrm{e}+2$ & 33 & 66 & $3.8 \mathrm{e}+2$ & 14 & 5.3 & $1.5 \mathrm{e}+2$ & 44 & 61 & $4.8 \mathrm{e}+2$ \\
\hline 121 & 40 & 15 & 6.2 & $1.1 \mathrm{e}+3$ & 35 & 83 & $2.5 \mathrm{e}+3$ & 16 & 6.6 & $1.1 \mathrm{e}+3$ & 49 & 77 & $3.6 \mathrm{e}+3$ \\
\hline
\end{tabular}

The convergence patterns reported in Table 1 and Table 2, showed that all preconditioners yielded scalable algorithms. When the number of nodes on each subdomain edge, $H / h$, was fixed and the number of subdomains, $N$, was increased, the iteration count showed only a slight growth. When $H / h$ was increased, while the partition was kept unchanged, the small increase in the number of iterations was satisfactory. The condition number estimates exhibited a similar dependence, or lack thereof, on $N$ and $H / h$. Note that the Dirichlet preconditioner for the DW setting, $M_{D}^{-1}$, albeit scalable, required the largest number of iterations, about three times as many as $F_{\text {gen }}^{-1}$, and had condition numbers about one order of magnitude larger than for $F_{\text {gen }}^{-1}$.

The generalized preconditioners $F_{\text {gen }}^{-1}$ and $\widetilde{M}_{\text {gen }}^{-1}$ had almost the same iteration counts and flop counts and were cheapest to implement. This was due in part to the fact that, for FETI-DP, the matrices $\operatorname{diag}\left(B_{r} B_{r}^{T}\right)$ and $\operatorname{diag}\left(B_{r} \widetilde{B}_{r}^{T}\right)$ were block diagonal. The Dirichlet preconditioner for mortars, $F_{D}^{-1}$, was noticeably more efficient than its DW counterpart, $M_{D}^{-1}$.

By comparing the convergence results from Table 1 to those from Table 2 for each preconditioner, we concluded that the answer to the question from the title of the paper is that choosing nonmortars does not influence the performance of the FETI-DP algorithms. A relatively small improvement in terms of iteration counts was achieved consistently for $M_{D}^{-1}$, the preconditioner for which a tighter condition number estimate was proved for the Neumann-Dirichlet choice of nonmortars in Dryja and Widlund [2002a]. 
The generalized mortar preconditioners $F_{\text {gen }}^{-1}$ and $\widetilde{M}_{\text {gen }}^{-1}$ performed very similarly and were clearly better in terms of iteration and flop counts and condition number estimates than the Dirichlet preconditioners $F_{D}^{-1}$ and $M_{D}^{-1}$.

We conclude by investigating the robustness of the FETI-DP algorithms for mortar discretizations in a more complicated setting, e.g., for an elliptic problem with jump coefficients, $-\operatorname{div}(\rho(x) \nabla u)=f$. The domain $\Omega$ was partitioned into four equal squares and $\rho(x)$ was chosen to be constant in each of these squares. The ratio of the constants in neighboring squares was 1000 . The mortar discretizations considered were similar to those used previously.

The results reported in Table 3 confirm the scalability of the FETI-DP algorithms with respect to the number of subdomains and to the number of nodes on each edge, for the generalized and Dirichlet preconditioners, modified as suggested in Klawonn et al. [2002] to account for the jump coefficients, for randomly chosen mortars. As expected, the generalized preconditioner performs better in terms of both condition numbers and computational costs.

Due to space constraints, we do not present the numerical results for the DW-type preconditioners, or for a Neumann-Dirichlet choice of nonmortars, but we note that exactly the same type of convergence behavior as for the Poisson problem was observed for the elliptic problem with jump coefficients.

Table 3. Convergence results, coefficients with jumps

\begin{tabular}{|c|c|c|c|c|c|}
\hline & & \multicolumn{2}{|c|}{ Generalized } & \multicolumn{2}{|r|}{ Dirichlet } \\
\hline$N$ & $H / h$ & Iter & Cond Mflops & Iter & Cond Mflops \\
\hline 16 & 5 & 11 & $9.2 \quad 4.7 \mathrm{e}-1$ & 35 & $541.6 \mathrm{e}+0$ \\
\hline 16 & 10 & 13 & $10.82 .6 \mathrm{e}+0$ & 37 & $687.4 \mathrm{e}+0$ \\
\hline 16 & 20 & 14 & $12.11 .6 \mathrm{e}+1$ & 38 & $804.8 \mathrm{e}+1$ \\
\hline 16 & 40 & 15 & $13.31 .3 \mathrm{e}+2$ & 41 & $923.7 \mathrm{e}+2$ \\
\hline 36 & 5 & 12 & $9.61 .5 \mathrm{e}+0$ & 36 & $564.5 \mathrm{e}+0$ \\
\hline 36 & 10 & 14 & $11.37 .2 \mathrm{e}+0$ & 39 & $712.0 \mathrm{e}+1$ \\
\hline 36 & 20 & 15 & $12.94 .5 \mathrm{e}+1$ & 42 & $781.3 \mathrm{e}+2$ \\
\hline 36 & 40 & 16 & $13.63 .3 \mathrm{e}+2$ & 46 & $911.0 \mathrm{e}+3$ \\
\hline 64 & 5 & 14 & $9.94 .0 \mathrm{e}+0$ & 40 & $611.2 \mathrm{e}+1$ \\
\hline 64 & 10 & 15 & $12.11 .6 \mathrm{e}+1$ & 43 & $744.4 \mathrm{e}+1$ \\
\hline 64 & 20 & 17 & $13.49 .4 \mathrm{e}+1$ & 47 & $892.7 \mathrm{e}+2$ \\
\hline 64 & 40 & 19 & $13.97 .2 \mathrm{e}+2$ & 50 & $982.0 \mathrm{e}+3$ \\
\hline 128 & 5 & 14 & $10.31 .0 \mathrm{e}+1$ & 42 & $633.0 \mathrm{e}+1$ \\
\hline 128 & 10 & 15 & $12.03 .6 \mathrm{e}+1$ & 45 & $761.1 \mathrm{e}+2$ \\
\hline 128 & 20 & 17 & $13.52 .0 \mathrm{e}+2$ & 48 & $915.6 \mathrm{e}+2$ \\
\hline 128 & 40 & 19 & $13.91 .5 \mathrm{e}+3$ & 52 & $1003.7 \mathrm{e}+3$ \\
\hline
\end{tabular}


Acknowledgement. This work was supported in part by the National Science Foundation under Grant NSF-DMS-0103588 and by the Research Foundation of the City University of New York under Award PSC-CUNY 65463-00 34.

\section{References}

C. Bernardi, Y. Maday, and A. Patera. A new non conforming approach to domain decomposition: The mortar element method. In H. Brezis and J.-L. Lions, editors, Collège de France Seminar. 1994.

M. Dryja and W. Proskurowski. A FETI-DP method for the mortar discretization of elliptic problems with discontinuous coefficients. In R. K. et al., editor, Proceedings of the 15th International Conference on Domain Decomposition Methods, Berlin, 2004. Springer-Verlag.

M. Dryja and O. B. Widlund. A FETI-DP method for a mortar discretization of elliptic problems. In L. Pavarino and A. Toselli, editors, Proceedings of the ETH Workshop on DDM. Springer-Verlag, 2002a.

M. Dryja and O. B. Widlund. A generalized FETI-DP method for the mortar discretization of elliptic problems. In I. H. et al., editor, Fourteenth International Conference on Domain Decomposition Methods, pages 27-38. ddm.org, 2002b. Mexico, 2002.

C. Farhat, M. Lesoinne, P. Le Tallec, K. Pierson, and D. Rixen. FETI-DP: A dual-primal unified FETI method - part I: A faster alternative to the two-level FETI method. Technical Report U-CAS-99-15, University of Colorado at Boulder, 1999.

C. Farhat, M. Lesoinne, and K. Pierson. A scalable dual-primal domain decomposition method. Numer. Lin. Alg. Appl., 7:687-714, 2000.

H.-H. Kim and C.-O. Lee. A FETI-DP preconditioner for elliptic problems on nonmatching grids. Technical Report 02-9, KAIST, 2002.

A. Klawonn, O. B. Widlund, and M. Dryja. Dual-Primal FETI methods for three-dimensional elliptic problems with heterogeneous coefficients. SIAM J. Numer. Anal., 40:159-179, 2002.

C. Lacour and Y. Maday. Two different approaches for matching nonconforming grids: the mortar element method and the FETI method. BIT, 37: 720-738, 1997.

J. Mandel and R. Tezaur. On the convergence of a Dual-Primal substructuring method. Numer. Math., 88:543-558, 2001.

D. Stefanica. A numerical study of FETI algorithms for mortar finite element methods. SIAM J. Sci. Comp., 23(4):1135-1160, 2001.

D. Stefanica. FETI and FETI-DP methods for spectral and mortar spectral elements: A performance comparison. J. Sci. Comp., 17(1-4):629-637, 2002.

B. Wohlmuth. A mortar finite element method using dual spaces for the Lagrange multiplier. SIAM J. Numer. Anal., 38:989-1012, 2000. 